\section{Keratinocyte growth factor prevents intra-alveolar oedema in experimental lung isografts}

\author{
J. Sadovski*,\$, T. Kuchenbuch*,\$, C. Ruppert" , A. Fehrenbach ${ }^{\mp}$, M. Hirschburger*, \\ W. Padberg*, A. Günther ${ }^{\#}$, J.M. Hohlfeld ${ }^{+}$, H. Fehrenbach ${ }^{\top}$ and V. Grau*
}

ABSTRACT: Primary graft dysfunction, characterised by intra-alveolar oedema, is a major obstacle in pulmonary transplantation. The present study evaluates the potential of keratinocyte growth factor (palmiferin; $\triangle$ N23-KGF) for the prevention of oedema in lung transplants.

Intratracheal instillation of $5 \mathrm{mg} \cdot \mathrm{kg}^{-1} \Delta \mathrm{N} 23-\mathrm{KGF}$ was performed in Lewis rats on days 3 and 2 before explantation. Control animals obtained an equivalent volume of vehicle. Left lungs were isogeneically transplanted and the graft recipients were sacrificed 1 day later for stereological analysis of intra-alveolar oedema and bronchoalveolar lavage. The total protein and phospholipid content, as well as surfactant proteins, were measured. Surfactant activity was analysed with a pulsating bubble surfactometer.

In grafts from control treated donors, the fraction of intra-alveolar oedema amounted to $3.4 \pm 1.1 \%$ of the total parenchymal volume. Treatment of donor lungs with $\Delta$ N23-KGF reduced oedema to a fraction of $1.6 \pm 0.8 \%$. In the lavage fluid of pulmonary grafts from $\Delta$ N23-KGF-treated donors, the total protein content was decreased compared with vehicle-treated lung transplants, whereas phospholipids did not differ. The protein fraction contained increased amounts of surfactant protein-C after $\Delta$ N23-KGF treatment and surfactant function was improved.

Treatment of donor lungs with palifermin protects against intra-alveolar oedema formation upon transplantation. This effect appears to be mediated by an improved surfactant homeostasis.

KEYWORDS: Keratinocyte growth factor, lung, oedema, primary graft dysfunction, transplantation

$\mathbf{T}$ ransplantation is the only curative therapy for numerous patients suffering from endstage lung diseases. During the first 3 months post-transplantation, $\sim 13 \%$ of pulmonary allograft recipients die [1]. Primary lung graft dysfunction (PGD) and infections are the main reasons for this poor survival early after transplantation and both conditions frequently coexist $[1,2]$. According to a recently proposed consensus definition, PGD is characterised by hypoxaemia and radiographic evidence of diffuse alveolar infiltrates [3]. Histopathological correlates of this syndrome are nonspecific alveolar damage and lung oedema [4].

Donor treatment with keratinocyte growth factor (KGF; fibroblast growth factor 7) might help to prevent PGD in pulmonary graft recipients, because KGF protects the lung in various models of acute lung injury, including hyperoxia, bleomycin, acid aspiration, mechanical injury, radiation, infections and even graft versus host disease [5]. Comparable to PGD, these models of lung damage involve pulmonary oedema formation. Lung protection by KGF is mediated by several mechanisms: most importantly, KGF stimulates the proliferation of alveolar epithelial type II cells (AEIIs) and results in a pronounced AEII hyperplasia on days 2 and 3 after treatment [6, 7]. The barrier function of both the alveolar epithelium and the endothelium [8], as well as the capacity of fluid reabsorption from the alveolar space, is improved [9]. KGF increases surfactant protein and phospholipid levels in vitro and in vivo [10-12]. Donor treatment with exogenous surfactant has been shown to be effective in preventing PGD in experimental settings [13-15] as well as in a small clinical trial [16]. Stimulation of the endogenous surfactant system by the application of KGF appears to be an appealing approach for the prevention of lung transplantion-associated reperfusion injury.

The use of KGF or the more stable N-terminally truncated analogue $\Delta$ N23-KGF (palifermin) [17] in the context of transplantation of lungs from
AFFILIATIONS

*Laboratory of Experimental Surgery, Dept of General and Thoracic Surgery,

\#Dept of Internal Medicine, University of Giessen Lung Center Justus-Liebig-University Giessen, Giessen,

"Clinical Research Group "Chronic Airway Diseases", Clinic of Internal Medicine (Respiratory Medicine), Philipps University of Marburg, Marburg, and +Fraunhofer Institute of Toxicology and Experimental Medicine, Hannover, Germany

${ }^{\S}$ These authors contributed equally to the study.

CORRESPONDENCE

V. Grau, Laboratory of Experimental Surgery, Dept of General and Thoracic Surgery, University of Giessen Lung Center, Justus-LiebigUniversity Giessen, RudolfBuchheim-Str. 7, D-35385 Giessen, Germany.

Fax: 496419944709

E-mail:Veronika.Grau@

chiru.med.uni-giessen.de

Received:

January 312007

Accepted after revision:

September 132007

\section{SUPPORT STATEMENT}

The study was supported by the Deutsche Forschungsgemeinschaft (FE 287/6-1; Bonn, Germany).

STATEMENT OF INTEREST

Statements of interest for

H. Fehrenbach and J.M. Hohlfeld can be found at www.erj.ersjournals.com/ misc/statements.shtml

European Respiratory Journal Print ISSN 0903-1936 Online ISSN 1399-3003 
deceased donors is limited by the fact that the lungs are most effectively protected when treated with KGF 2-3 days before the injurious stimulus is applied. Lung protection by KGF might, however, be useful in living donor lobar lung transplantation that is performed in paediatric patients to mitigate the growing competition for deceased donor lungs [18]. As lung regeneration is also stimulated by KGF [19], both graft donor and recipient might profit from KGF application to donor lungs.

The aim of the present study was to evaluate the potential of donor lung treatment with $\Delta \mathrm{N} 23-\mathrm{KGF}$ in order to prevent alveolar oedema formation after transplantation. The current authors used an experimental rat model of isogeneic orthotopic left lung transplantation involving short ischaemic times comparable to the situation in living donor lung transplantation.

\section{MATERIALS AND METHODS}

\section{Study design}

Lung protection by KGF is only effective when the growth factor is applied 2-3 days before injury [5]. In contrast to living donor lungs, grafts from deceased donors can probably not be protected against PGD. Therefore, the current authors chose an experimental design resembling the living donor procedure, in which ischaemic times are kept as short as possible.

Inbred Lewis (LEW) donor rats were treated on days 1 and 2 with bioactive recombinant human $\Delta \mathrm{N} 23-\mathrm{KGF}$ or vehicle via intratracheal instillation. On day 4, orthotopic left lung transplantation to isogeneic recipients was performed. Warm and cold ischaemic times were kept as short as possible. Oedema formation was evaluated by stereological methods, by protein and surfactant analysis in the bronchoalveolar lavage (BAL) fluid $24 \mathrm{~h}$ after transplantation. This point in time was chosen because in this experimental model, intra-alveolar oedema was obvious 1 day after transplantation and resolved on days 2 and 3 [20].

\section{Animals}

Male LEW $\left(\mathrm{RT}^{l}\right)^{l}$ rats were raised under pathogen-free conditions according to Federation of European Laboratory Animal Science Associations standards and provided by Harlan Winkelmann (Borchen, Germany). Animal care and animal experiments were performed following the current version of the German Law on the Protection of Animals as well as the National Institutes of Health principles of laboratory animal care.

\section{KGF-treatment and lung transplantation}

Bioactive, purified, endotoxin-free $\Delta$ N23-KGF (palifermin) produced in Escherichia coli was provided by Amgen (Thousand Oaks, CA, USA). On day 1, animals weighing 200-270 g were anaesthetised by short time inhalation of isofluran (Forene; Abbot, Wiesbaden, Germany), intubated orally with a 16G intravascular catheter (Insyte; Becton Dickinson, Sandy, UT, USA), and instilled via the trachea with $5 \mathrm{mg} \Delta \mathrm{N} 23-\mathrm{KGF}$ per $\mathrm{kg}$ body weight or an equivalent volume (200-270 $\mu \mathrm{L}$ ) of PBS. The treatment was repeated on day 2. On day 4 , these rats served as donors for lung transplantation.

Orthotopic left lung transplantation was performed in the isogeneic LEW to LEW rat strain combination as described
[20]. In brief, a cuff technique was used for the vascular anastomoses and sutures with interrupted stitches for the bronchial anastomoses. Warm ischaemic times remained $<20 \mathrm{~min}$ and cold ischaemic times $<25 \mathrm{~min}$. No immunosuppression was given.

Pulmonary grafts were harvested $24 \mathrm{~h}$ after transplantation for histology or BAL. Only grafts which were ventilated and supplied with blood were considered to be technically successful and included in the study.

\section{Graft histology}

The lungs were flushed in situ with $20 \mathrm{~mL}$ saline via the pulmonary artery and fixed by vascular perfusion with $1.5 \%$ glutaraldehyde, $1.5 \%$ paraformaldehyde dissolved in $0.15 \mathrm{M}$ HEPES buffer, $\mathrm{pH} 7.4$, at a hydrostatic pressure of $15 \mathrm{cmH}_{2} \mathrm{O}$ $(1.5 \mathrm{kPa})$ for $20 \mathrm{~min}$ while the airway pressure was adjusted to $12 \mathrm{cmH}_{2} \mathrm{O}(1.2 \mathrm{kPa})$. Thereafter, the trachea was ligated and the heart-lung block was removed from the recipient rat. Fixation by perfusion was followed by $24 \mathrm{~h}$ immersion in the same fixative at $4^{\circ} \mathrm{C}$.

After determination of the lung volume by fluid displacement, lungs were embedded into $2 \%$ aqueous agar-agar at $40^{\circ} \mathrm{C}$, cooled to room temperature, stored at $8^{\circ} \mathrm{C}$ for $2-3 \mathrm{~h}$, and then cut into $4-\mathrm{mm}$ thick slices by means of a tissue slicer, with the first cut being at a random position within the first $4 \mathrm{~mm}$. The slices were post-fixed with $1 \%$ osmium tetroxide in $0.1 \mathrm{M}$ sodium cacodylate buffer followed by overnight incubation in half-saturated aqueous uranyl acetate. Slices were dehydrated through a series of graded acetone, and embedded into glycol methacrylate (Technovit 7100; Kulzer, Wehrheim, Germany). After staining of semi-thin sections with methylene blue/Azur II, intra-alveolar oedema was determined by stereological analysis.

\section{Stereological analysis}

Intra-alveolar oedema was determined by point counting according to a two-level cascade sampling procedure [21] using a light microscope (Olympus BX51; Olympus, Copenhagen, Denmark) equipped with a computer-assisted stereology toolbox (CAST Grid; Visiopharm, Hersholm, Denmark). At level 1, the volume densities of nonparenchyma (airways, arteries, veins and pleura) and parenchyma (alveolar septa, airspace of alveoli and alveolar ducts) were estimated at a primary magnification of $4 \times$. At level 2 , the volume densities of alveolar and alveolar duct airspace (occupied by air or oedema fluid), alveolar septal tissue and capillary lumen were estimated at a primary magnification of $20 \times$. At each level, a set of systematic uniform random sampled fields of view were collected for point counting. Volume densities, fractions of total lung volume, and absolute volumes of each compartment were calculated as described previously [22]. Absolute volumes of the transplants and the right native lungs were normalised to body weight of the donors and the recipient rats, respectively, and are given as mass-specific volumes.

\section{$B A L$}

Pulmonary graft recipients were anaesthetised and the trachea was cannulated. After sternotomy, the right main bronchi were clamped and the left transplanted lungs were each lavaged five times with $2 \mathrm{~mL}$ saline at $37^{\circ} \mathrm{C}$. Thereafter, the right lungs were 

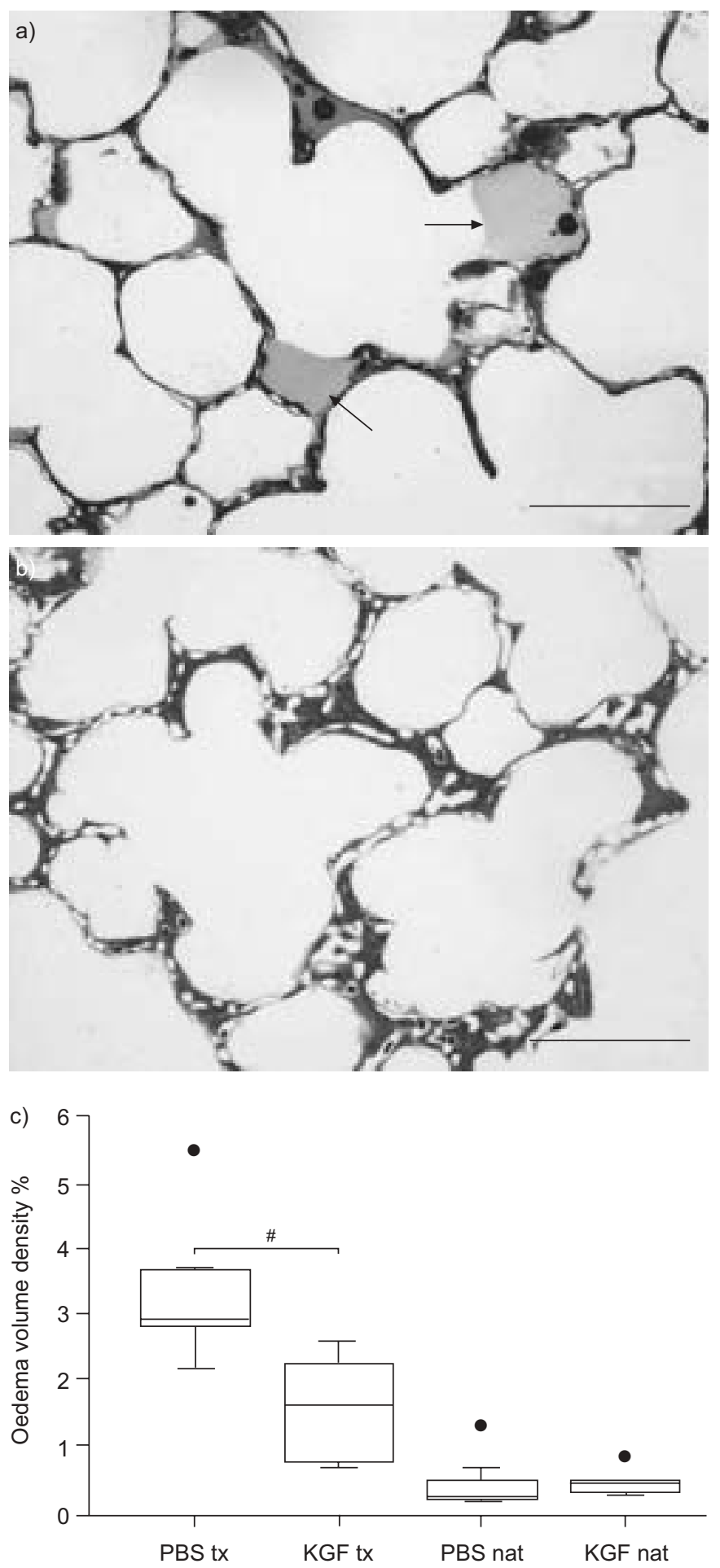

FIGURE 1. Histopathology of pulmonary isografts 1 day after transplantation. a) Semi-thin section of the lung parenchyma of a PBS-treated transplant stained with methylene blue/Azur II. The arrows indicate intra-alveolar oedema. b) Section of a pulmonary transplant which was pre-treated with keratinocyte growth factor (KGF; $\Delta$ N23-KGF). The alveolar walls are thickened due to alveolar epithelial type II cell hyperplasia. Intra-alveolar oedema is rare. c) Volume fraction (\% total parenchyma) of the intra-alveolar oedema in lung transplants (tx) and right native lungs (nat) after PBStreatment $(n=6)$ or treatment with $\triangle N 23-K G F(n=7)$. The box plots indicate median and percentiles $0,25,75$ and 100 . @: data beyond $\pm 3 \times$ SD. ${ }^{\#}: p \leqslant 0.01$. Scale bars $=100 \mu \mathrm{m}$ each lavaged five times with $3 \mathrm{~mL}$ saline. BAL fluid (BALF) was collected on ice, centrifuged for $15 \mathrm{~min}$ at $300 \times g$ and stored at $-20^{\circ} \mathrm{C}$ until further analysis.

\section{Analysis of BALF}

Total proteins were quantified in BALF using a commercial assay kit (BCA; Pierce, Bonn, Germany). The content of the hydrophobic surfactant proteins (SP)-B and SP-C in the BALF was determined by ELISA as previously described [23, 24].

BALF was centrifuged at $48,000 \times g\left(1 \mathrm{~h}, 4^{\circ} \mathrm{C}\right)$ using a Beckmann L5-50 centrifuge (type 40 rotor; Beckmann Coulter $\mathrm{GmbH}$, Krefeld, Germany) to isolate large surfactant aggregates (LA). The LA-containing pellets were resuspended in $0.9 \% \mathrm{NaCl}$ and the relative LA content was assessed by relating the fraction of pelleted phospholipid to the total amount of phospholipid. Lipids were extracted from original BALF or the isolated LA fraction with chloroform/methanol according to the method of BLIGH and DYER [25], and organic phases were taken for quantification. Phospholipids were quantified by means of a colorimetric phosphorus assay as previously described [26].

\section{Measurement of surfactant surface activity}

Surface activity of surfactant material was measured with a pulsating bubble surfactometer [27]. Experiments were performed at hypophase concentrations of $1 \mathrm{mg}$ phospholipids per mL. Briefly, $40 \mu \mathrm{L}$ of the resuspended LA pellet were injected into the bubble chamber. Before starting bubble pulsation the material was allowed to stabilise for $5 \mathrm{~min}$. Measurements were performed with a cycle speed of 20 cycles $\cdot \mathrm{min}^{-1}$ for $5 \mathrm{~min}$. Prior to bubble oscillation, phospholipid adsorption $\left(\gamma_{\text {ads }}\right)$ was measured as the surface tension of the originated bubble after a time period of $10 \mathrm{~s}$. Minimum surface tension at minimum radius of the bubble after 5 min of pulsation was defined as $\gamma_{\text {min }}$. Similarly, maximum surface tension at maximum radius was defined as $\gamma_{\max }$. Data were digitalised and recorded by computer.

\section{Statistical analysis}

Data are given as mean \pm SD in the text, are presented in box plots in graphs, and were analysed with nonparametric Kruskal-Wallis test followed by Mann-Whitney rank sum test, with $p \leqslant 0.05$ set as the significance level.

\section{RESULTS}

\section{Transplantation of 4 N23-KGF-treated lungs}

LEW donor rats were treated on days 1 and 2 with $\triangle$ N23-KGF via intratracheal instillation, control donors obtained an equivalent volume of PBS. Lungs were transplanted on day 4 and harvested $24 \mathrm{~h}$ thereafter. The technical success rate was identical in both experimental groups: $86 \%$ for PBS-treated donors $(n=28)$ and $85 \%$ for $\Delta$ N23-KGF-treated donors $(n=40)$.

\section{Graft histopathology}

In transplants from PBS-treated donors, intra-alveolar oedema could be detected (fig. 1a). Alveoli containing protein-rich oedema fluid formed clusters surrounded by unimpaired lung parenchyma. Frequently, a quarter to a half of the alveolar area was filled with oedema, which lined the alveolar wall (fig. 1a). However, no severe damage of the lung parenchyma was seen. As described previously for lung isografts on day 1 


\begin{tabular}{|c|c|c|c|}
\hline TABLE 1 & \multicolumn{3}{|c|}{$\begin{array}{l}\text { Results of stereological analysis of transplanted } \\
\text { left lungs }\end{array}$} \\
\hline \multicolumn{2}{|l|}{ Parameters } & PBS treated & $\Delta$ N23-KGF treated \\
\hline \multicolumn{2}{|l|}{ Subjects $\mathrm{n}$} & 7 & 6 \\
\hline \multicolumn{2}{|c|}{ Body weight g } & $239.0 \pm 2.2$ & $259.0 \pm 14.0^{\#}$ \\
\hline \multicolumn{4}{|c|}{$\begin{array}{l}\text { Mass-specific volumes } \\
\mathrm{mm}^{3} \cdot \mathrm{g}^{-1} \text { body weight }\end{array}$} \\
\hline \multicolumn{2}{|l|}{ Lung } & $11.1 \pm 3.0$ & $12.7 \pm 1.1$ \\
\hline \multicolumn{2}{|c|}{ Nonparenchyma } & $1.3 \pm 0.5$ & $1.4 \pm 0.5$ \\
\hline \multicolumn{2}{|c|}{ Parenchyma } & $9.8 \pm 2.5$ & $11.3 \pm 1.1$ \\
\hline \multicolumn{2}{|c|}{ Air space } & $8.2 \pm 2.2$ & $9.6 \pm 1.0$ \\
\hline \multicolumn{2}{|c|}{ Alveolar oedema } & $0.32 \pm 0.11$ & $0.18 \pm 0.10^{\#}$ \\
\hline \multicolumn{2}{|c|}{ Septal tissue } & $0.86 \pm 0.35$ & $1.12 \pm 0.17$ \\
\hline \multicolumn{2}{|c|}{ Capillary lumen } & $0.38 \pm 0.13$ & $0.42 \pm 0.15$ \\
\hline
\end{tabular}

Data are presented as mean $\pm S D$, unless otherwise stated. $\triangle \mathrm{N} 23-\mathrm{KGF}$ : $\mathrm{N}$ terminally truncated keratinocyte growth factor analogue (palifermin). ${ }^{*}: p \leqslant 0.05$.

post-transplantation, the connective tissue surrounding blood vessels exhibited oedematous swelling and contained some mononuclear leukocytes [20].

Lung isografts originating from donors that were treated with $\triangle \mathrm{N} 23-\mathrm{KGF}$ (palifermin) revealed pronounced hyperplasia of AEIIs (fig. 1b). This is a typical feature of rat lungs treated with KGF, indicating that the treatment was successful [5]. In comparison to control pulmonary transplants, the area of the intra-alveolar space filled with oedema was reduced (fig. 1b). The perivascular oedema and leukocytic infiltrate in KGFtreated pulmonary isografts did not differ from PBS-treated isografts.

\section{Stereological analysis of intra-alveolar oedema}

To quantify the intra-alveolar oedema in vehicle-treated and KGF-treated lungs, stereological analysis was performed (table 1; fig. 1c).

\begin{tabular}{lcc}
\hline TABLE 2 & $\begin{array}{c}\text { Results of stereological analysis of native right } \\
\text { recipient lungs }\end{array}$ \\
Parameters & $\begin{array}{c}\text { Recipient of } \\
\text { PBS-treated lung }\end{array}$ & $\begin{array}{c}\text { Recipient of } \mathbf{\Delta N 2 3 - K G F - ~} \\
\text { treated lung }\end{array}$ \\
\hline Subjects $\mathbf{n}$ & 7 & 6 \\
Body weight g & $241.1 \pm 1.4$ & $258.3 \pm 5.5^{\#}$ \\
Mass-specific volumes & & \\
mm $^{3} \cdot \mathbf{g}^{-1}$ body weight & & \\
Lung & $18.7 \pm 4.7$ & $22.3 \pm 4.2$ \\
Nonparenchyma & $2.3 \pm 0.4$ & $2.3 \pm 0.6$ \\
Parenchyma & $16.3 \pm 4.5$ & $20.1 \pm 3.9$ \\
$\quad$ Air space & $14.6 \pm 4.4$ & $17.5 \pm 3.6$ \\
$\quad$ Alveolar oedema & $0.06 \pm 0.06$ & $0.08 \pm 0.05$ \\
Septal tissue & $1.2 \pm 0.2$ & $1.6 \pm 0.4$ \\
Capillary lumen & $0.6 \pm 0.1$ & $0.8 \pm 0.4$ \\
\hline
\end{tabular}

Data are presented as mean \pm SD, unless otherwise stated. $\triangle$ N23-KGF: Nterminally truncated keratinocyte growth factor analogue (palifermin). ${ }^{\#}: p \leqslant 0.05$.
In isografts originating from PBS-treated donors, the fraction of intra-alveolar oedema amounted to $3.4 \pm 1.1 \%$ of total parenchymal volume. Pre-treatment of donor lungs with $\Delta$ N23KGF significantly $(\mathrm{p}=0.005)$ reduced the oedema to a fraction of $1.6 \pm 0.8 \%$ (fig. 1c). Relating intra-alveolar oedema volume to the alveolar air-blood barrier, oedema amounts to $41.1 \pm 17.8 \%$ of tissue volume in transplanted lungs of PBS-treated rats versus $16.6 \pm 9.8 \%$ after $\Delta \mathrm{N} 23-K G F-$ treatment $(\mathrm{p}=0.005)$. Massspecific absolute oedema volume was also significantly reduced (table 1). The native right lung was included in the present analysis as an internal control (table 2). No differences were observed between the right native lungs from recipients of PBS-treated or $\triangle$ N23-KGF-treated left lungs.

\section{Analysis of BALF}

PBS-treated or $\triangle \mathrm{N} 23-K G F-$ treated grafts and the native right lung were lavaged separately. In addition, lungs from sex- and age-matched healthy LEW rats were included.

Transplantation of PBS-treated lungs resulted in a significant $(\mathrm{p} \leqslant 0.01)$ increase in the total protein content in the BALF from $2.9 \pm 0.2 \mathrm{mg}$ per left lung in healthy controls to $12.9 \pm 4.2 \mathrm{mg}$ per graft (fig. 2). Application of $\Delta \mathrm{N} 23-\mathrm{KGF}$ before transplantation reduced the amount of protein to $6.4 \pm 1.4 \mathrm{mg}$ per graft $(p \leqslant 0.01)$. The protein content of the right native lungs of all experimental groups remained at about the same level.

SP-B and SP-C were measured in the BALF by ELISA. The total SP-B content in BALF from left control lungs, PBS-treated grafts and $\Delta$ N23-KGF-treated grafts was identical. The same was true for the right native lungs. Due to the increase in the total protein and phospholipid content upon lung transplantation, the SP-B/total protein ratio decreased, but no significant differences were obvious between PBS-treated and $\triangle$ N23-KGFtreated pulmonary isografts (fig. 2).

In contrast, the total SP-C content in BALF was higher in grafts compared with healthy control left lungs $(p \leqslant 0.05)$. No significant difference was seen among PBS-treated and $\Delta 23$ KGF-treated grafts, or among the right lungs from all experimental groups. As expected, the SP-C/total protein ratio was decreased $(p \leqslant 0.05)$ in PBS-treated transplants. However, in BALF obtained from $\triangle$ N23-KGF-treated grafts the SP-C/ total protein ratio did not differ from native control lungs, and was higher $(p \leqslant 0.01)$ when compared with the ratios measured in PBS-treated grafts (fig. 2).

Transplantation increased the amount of phospholipids harvested from the left lung by BAL $(p \leqslant 0.01)$. Among the right native lungs no changes were seen. $\Delta$ N23-KGF-treatment did not influence the phospholipid content in comparison to PBStreated grafts. Consequently, the phospholipid/protein ratio decreased $(p \leqslant 0.01)$ in PBS-treated pulmonary transplants, whereas in isografts from $\Delta \mathrm{N} 23-\mathrm{KGF}$-treated donors, this ratio did not differ from healthy control lungs (fig. 2). The difference between PBS-treated grafts and $\triangle$ N23-KGF-treated grafts was statistically significant $(\mathrm{p} \leqslant 0.05)$. LA content was slightly increased in $\triangle$ N23-KGF-treated lungs, but did not statistically differ from PBS-treated lungs (93.2 \pm 11.0 versus $82.2 \pm 15.6)$.

\section{Surfactant activity}

Surfactant activity was measured with a pulsating bubble surfactometer. The results are summarised in table 3 . The 

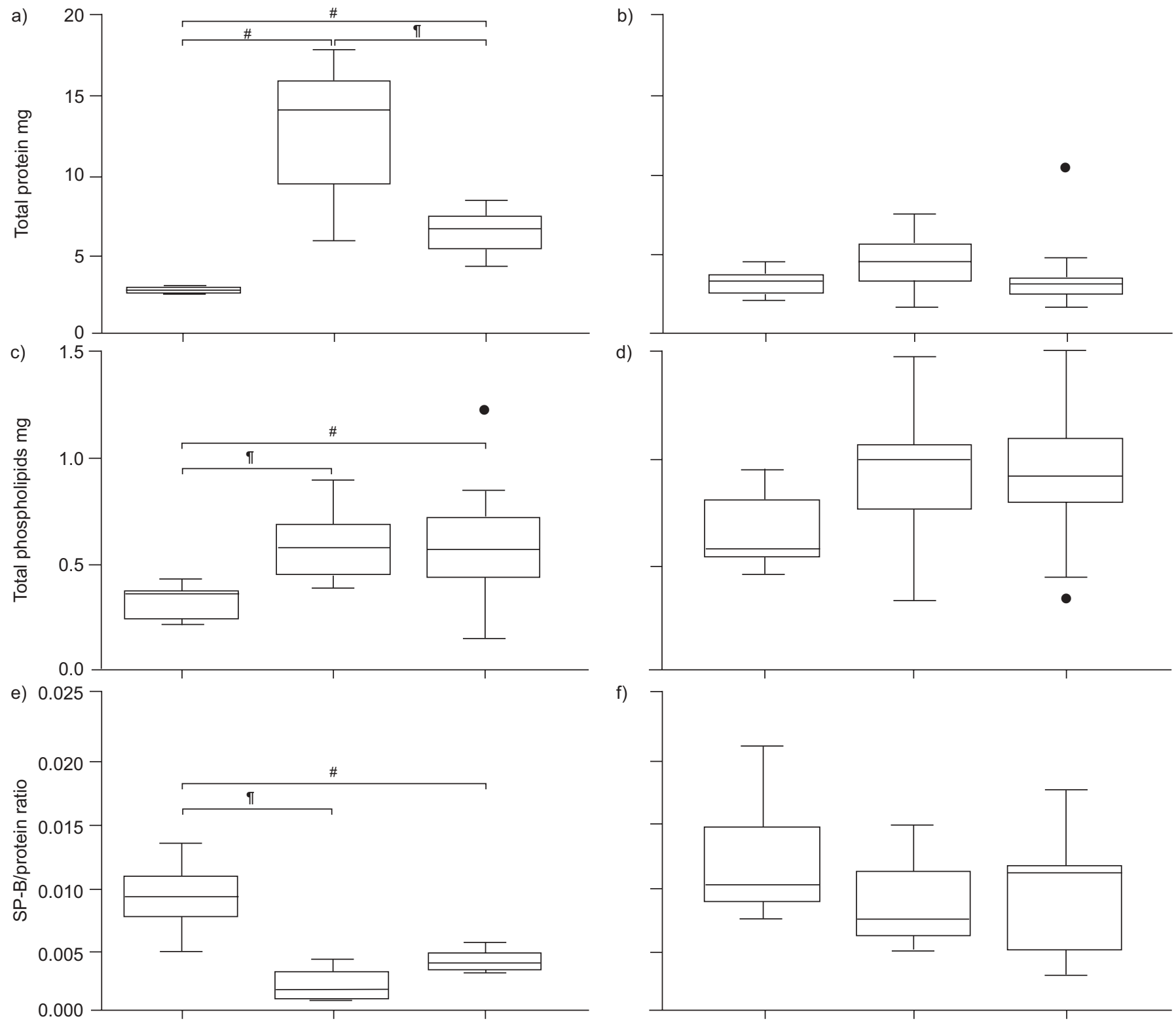

d)

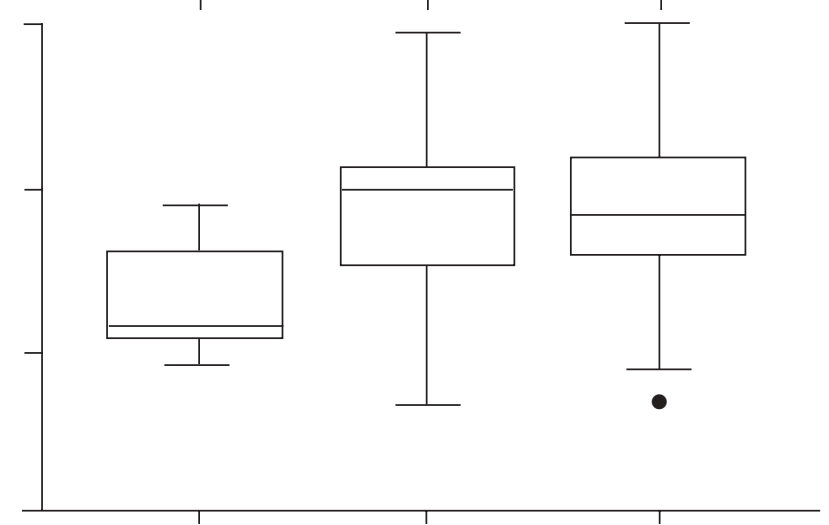

f)

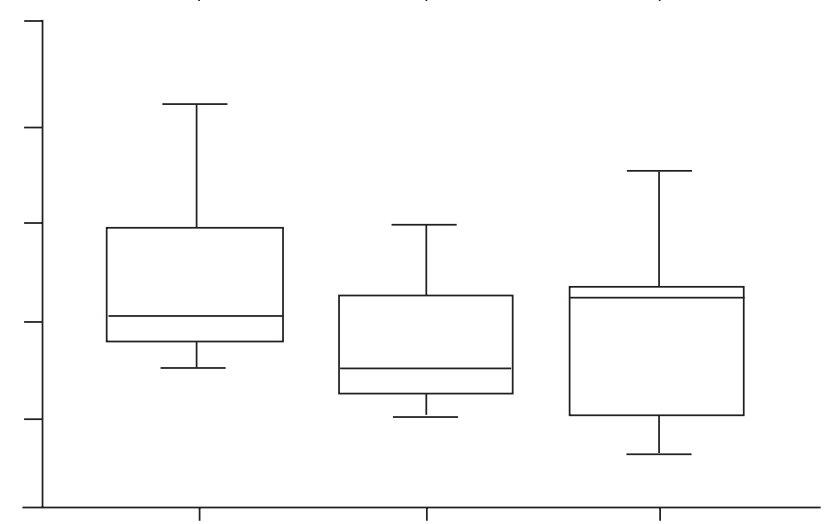

h)

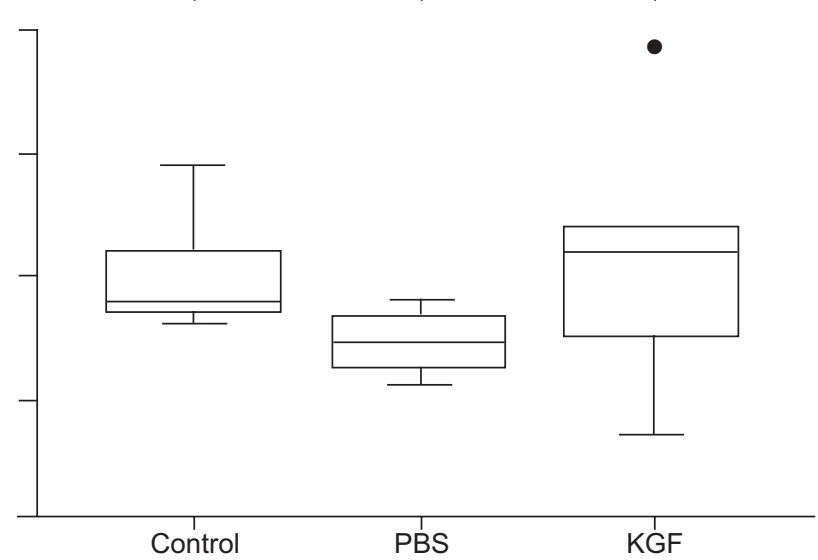

FIGURE 2. Analysis of bronchoalveolar lavage (BAL) fluid of the left lungs $(a, c, e, g)$ and the right lungs $(b, d, f, h)$ of healthy controls and recipients of pulmonary isografts. Left lungs were transplanted and the right native lung of the recipients remained in the recipient. The total protein and phospholipid content in the BAL was determined, as well as the ratio of surfactant protein (SP)-B or SP-C to the total protein content. The box plots indicate median and percentiles $0,25,75$ and 100 . a-d) The numbers of observations were: control $n=5$; PBS $n=9$; and keratinocyte growth factor (KGF) $n=10$. e-h) The numbers of observations were: control $n=5$; PBS $n=4$; and KGF $n=5$. •: data beyond $\pm 3 \times S$ D. ${ }^{\#}: p \leqslant 0.05 ;{ }^{\circ}: p \leqslant 0.01$. 
surface tension $\left(\gamma_{\text {ads }}, \gamma_{\min }\right.$ and $\left.\gamma_{\max }\right)$ of the isolated surfactant tended to be reduced in $\Delta$ N23-KGF-treated transplants in comparison with PBS-treated grafts. This difference was statistically significant $(\mathrm{p} \leqslant 0.05)$ for $\gamma_{\max }$. A similar effect was also seen for the right native lung of recipients of $\triangle$ N23-KGFtreated lungs in comparison to PBS-treated lungs.

\section{DISCUSSION}

The present study demonstrated that lungs from $\Delta$ N23-KGFtreated donor rats can be successfully transplanted. In addition, treatment of donor lungs with $\Delta$ N23-KGF protects against perioperative lung injury, which inevitably occurs during transplantation.

Impaired function of the air-blood barrier, resulting in the formation of intra-alveolar oedema is a hallmark of PGD [2]. Protection against PGD in lung grafts from $\triangle$ N23-KGF-treated donors compared with PBS-treated grafts was demonstrated in the present study by independent techniques. First, a stereological approach was chosen for quantification of lung oedema in lungs fixed by vascular perfusion, because this technique is more sensitive when compared with the analysis of the wet/ dry weight ratio [22]. Sensitivity is important, because in the present experimental setting involving short ischaemic times the amount of intra-alveolar oedema is low. Furthermore, stereological data on lung oedema, but not wet/dry weight ratio data, have been shown to significantly correlate with lung function [22].

In a second approach, the current authors measured the protein content in the BALF. According to published data [13], BALF from PBS-treated lung transplants contained more protein compared with healthy control lungs. However, donor $\Delta$ N23-KGF-treatment resulted in a significantly decreased protein content in the BALF of lung transplants.

A direct functional analysis of the lung transplants was not performed because the volume density of the intra-alveolar oedema in PBS-treated grafts was too small to expect a severe impairment of blood oxygenation in comparison with $\Delta \mathrm{N} 23$ KGF-treated lungs. A previous study investigating the effects of various preservation solutions on ischaemia/reperfusion

\begin{tabular}{lccc} 
TABLE 3 & \multicolumn{3}{c}{$\begin{array}{l}\text { Surface activity of surfactant isolated from } \\
\text { pulmonary transplants }(\mathrm{tx}) \text { and native lungs (nat) }\end{array}$} \\
& $\gamma_{\text {ads }} \mathbf{~} \mathbf{~ N} \cdot \mathbf{m}^{-1}$ & $\gamma_{\mathbf{m i n}} \mathbf{~} \mathbf{N} \cdot \mathbf{m}^{-1}$ & $\gamma_{\max } \mathbf{~} \mathbf{N} \cdot \mathbf{m}^{-1}$ \\
\hline PBS tx & $36.5 \pm 10.7$ & $23.6 \pm 8.8$ & $45.2 \pm 7.0$ \\
KGF tx & $28.2 \pm 5.2$ & $18.1 \pm 1.2$ & $35.2 \pm 3.4^{\#}$ \\
PBS nat & $30.8 \pm 3.9$ & $16.9 \pm 2.2$ & $40.1 \pm 3.1$ \\
KGF nat & $28.5 \pm 9.0$ & $11.5 \pm 5.7$ & $32.3 \pm 4.2^{*}$ \\
\hline
\end{tabular}

Data are presented as mean \pm SD and $n=4$ for all observations. Graft donors were either vehicle-treated (PBS) or treated with $\mathrm{N}$-terminally truncated keratinocyte growth factor analogue (KGF). $\gamma_{\text {ads }}$ : phospholipid adsorption; $\gamma_{\text {min }}$ : minimum surface tension at minimum radius of the bubble after $5 \mathrm{~min}$ of pulsation; $\gamma_{\max }$ : maximum surface tension at maximum radius. ${ }^{\#}$ : statistically significant difference between KGF-treated and PBS-treated lungs $p \leqslant 0.05$; statistically significant difference between the right native lungs of recipients of KGF-treated and PBS-treated lungs $p \leqslant 0.05$. injury demonstrated that the oxygen tension measured during reperfusion was largely normal in rat lungs exhibiting a volume density of intra-alveolar oedema of up to $3 \%$, but dropped sharply beyond 3\% [22]. Although functional impairment cannot be inferred as being severe in PBS-treated grafts ( $3.4 \%$ oedema), it can clearly be expected to be worse than in KGF-treated grafts (1.6\% oedema). However, such differences might be obscured by the minor functional impairments that were observed after KGF-treatment [28].

A large body of literature describes improved surfactant homeostasis after lung treatment with KGF and implicates that, at least in part, protective effects of KGF are mediated via surfactant $[5,29]$. Surfactant function is impaired during the reimplantation response in rat lung transplants and instillation of surfactant improves early graft function [13]. Beneficial effects of exogenous surfactant application were additionally shown in mini-pigs [15], dogs [14] and in a small clinical study in human transplant patients [16]. Therefore, the current authors analysed the total phospholipid content of the BALF, as well as SP-B and SP-C, small hydrophobic proteins that are most important in terms of biophysical functions of surfactant [30].

In line with previous studies [13], the present results demonstrate that some characteristic parameters of pulmonary surfactant were changed upon lung transplantation in the experimental model: the total phospholipid content and the content of SP-C were increased in response to transplantation, whereas SP-B remained unchanged. However, the differences in the total amounts of these components were not significant between grafts from PBS- and $\Delta$ N23-KGF-treated donors, which may relate to the fact that the degree of injury was low in the experiments. The only parameter related to surfactant proteins which differed significantly was the SP$\mathrm{C} /$ total protein ratio, which might be functionally relevant because SP-C increases the resistance of surfactant to inhibition by serum proteins or oedema fluid [30].

Surfactant from $\Delta$ N23-KGF-treated pulmonary grafts tended to be more active compared with PBS-treated grafts, with $\gamma_{\max }$ being significantly different. In contrast to the protein content of the BAL fluid, surfactant function was also reduced in the right native lungs of graft recipients, which might result from mechanical ventilation during surgery. In addition, the pulmonary graft appeared to contribute to the impairment of reduction function in the right native lung, since protection of the graft with $\triangle \mathrm{N} 23-\mathrm{KGF}$ resulted in a better surfactant function in the native lung. This might be explained by a functional overload of the native lung in the presence of a damaged graft.

The time schedule to achieve effective lung protection by KGF-treatment has been carefully optimised previously by other authors [5]. Donor KGF-treatment 2-3 days before transplantation seems to be mandatory when aiming at a prevention of PGD. Therefore, KGF-treatment appears to be limited to living donor lobar lung transplantation. However, pulmonary allografts are additionally damaged by postoperative mechanical ventilation, infections and rejection. Further experimental studies will be needed to evaluate the protective role of KGF against these injuries. If so, recipients of lungs from deceased donors might also benefit from KGF application in the perioperative or post-operative phase. 
In conclusion, donor lung pre-treatment with palifermin reduced intra-alveolar oedema, the histopathological correlate of primary lung graft dysfunction, in the rat lung transplantation model. In living donor lung transplantation, palifermin treatment might be beneficial for donor and recipient, as keratinocyte growth factor has been evidenced to promote lung regeneration upon partial pneumonectomy [19]. The current authors suggest that donor lung treatment with palifermin might be a promising strategy for the prevention of early graft dysfunction in humans, particularly when living donor transplantations are performed.

\section{ACKNOWLEDGEMENTS}

The authors would like to thank S. Iffländer, who cared for the experimental animals and U. Berges (both Justus-LiebigUniversity, Giessen, Germany) for help with the illustrations. The authors are also grateful for the expert technical assistance of R. Plass, P. Freitag (both Justus-Liebig-University, Giessen, Germany), R. Naumann and T. Rausch (both PhillippsUniversity, Marburg, Germany).

\section{REFERENCES}

1 Trulock EP, Edwards LB, Taylor DO, et al. Registry of the International Society for Heart and Lung Transplantation: twenty-third official adult lung and heart-lung transplantation report - 2006. J Heart Lung Transplant 2006; 25 : 880-892.

2 Wilkes DS, Egan TM, Reynolds HY. Lung transplantation: opportunities for research and clinical advancement. Am J Respir Crit Care Med 2005; 172: 944-955.

3 Christie JD, Van Raemdonk D, de Perrot M, et al. Report of the ISHLT Working Group on Primary Lung Graft Dysfunction part I: introduction and methods. I Heart Lung Transplant 2005; 24: 1451-1453.

4 de Perrot M, Liu M, Waddel TK, Keshavjee S. Ischemiareperfusion-induced lung injury. Am J Respir Crit Care Med 2003; 167: 490-511.

5 Ware LB, Matthay MA. Keratinocyte and hepatocyte growth factors in the lung: roles in lung development, inflammation, and repair. Am J Physiol Lung Cell Mol Physiol 2002; 282: L924-L940.

6 Ulich TR, Yi ES, Longmuir K, et al. Keratinocyte growth factor is a growth factor for type II pneumocytes in vivo. J Clin Invest 1994; 93: 1298-1306.

7 Fehrenbach H, Kasper M, Tschernig T, et al. Keratinocyte growth factor-induced hyperplasia of rat alveolar type II cells in vivo is resolved by differentiation into type I cells and by apoptosis. Eur Respir J 1999; 14: 534-544.

8 Barazzone C, Donati YR, Rochat AF, et al. Keratinocyte growth factor protects alveolar epithelium and endothelium from oxygen-induced injury in mice. Am J Pathol 1999; 154: 1479-1487.

9 Guery BP, Mason CM, Dobard EP, Beaucaire G, Summer WR, Nelson S. Keratinocyte growth factor increases transalveolar sodium reabsorption in normal and injured rat lungs. Am J Respir Crit Care Med 1997; 55: 1777-1784.

10 Sugahara K, Rubin JS, Mason RJ, Aronsen EL, Shannon JM. Keratinocyte growth factor increases mRNAs for SP-A and
SP-B in adult rat alveolar type II cells in culture. Am J Physiol 1995; 269: L344-L350.

11 Morikawa O, Walker TA, Nielsen LD, Pan T, Cook JL, Mason RJ. Effect of adenovector-mediated gene transfer of keratinocyte growth factor on the proliferation of alveolar type II cells in vitro and in vivo. Am J Respir Cell Mol Biol 2000; 23: 626-635.

12 Yano T, Mason RJ, Pan T, Deterding RR, Nielsen LD, Shannon JM. KGF regulates pulmonary epithelial proliferation and surfactant protein gene expression in adult rat lung. Am J Physiol Lung Cell Mol Physiol 2000; 279: L1146L1158.

13 Erasmus ME, Petersen AH, Hofstede G, Haagsman HP, Bambang Oetomo S, Prop J. Surfactant treatment before reperfusion improves the immediate function of lung transplants in rats. Am J Respir Crit Care Med 1996; 153 665-670.

14 Günther A, Balser M, Schmidt R, et al. Surfactant abnormalities after single lung transplantation in dogs: impact of bronchoscopic surfactant administration. J Thorac Cardiovasc Surg 2004; 127: 344-354.

15 Hohlfeld JM, Struber M, Ahlf K, et al. Exogenous surfactant improves survival and surfactant function in ischaemiareperfusion injury in minipigs. Eur Respir J 1999; 13: 1037-1043.

16 Strüber M, Hirt SW, Cremer J, Harringer W, Haverich A. Surfactant replacement in reperfusion injury after clinical lung transplantation. Intensive Care Med 1999; 25: 862-864.

17 Hsu E, Osslund T, Nybo R, et al. Enhanced stability of recombinant keratinocyte growth factor by mutagenesis. Protein Eng Des Sel 2006; 19: 147-153.

18 Sweet SC. Pediatric living donor lobar lung transplantation. Pediatr Transplant 2006; 10: 861-868.

19 Kaza AK, Kron IL, Leuwerke SM, Tribble CG, Laubach VE. Keratinocyte growth factor enhances post-pneumonectomy lung growth by alveolar proliferation. Circulation 2002; 106: I120-I124.

20 Schmidt A, Sucke J, Fuchs-Moll G, et al. Macrophages in experimental lung isografts and allografts: infiltration and proliferation in situ. J Leukocyte Biol 2007; 81: 186-194.

21 Ochs M. A brief update on lung stereology. J Microsc 2006; 222: 188-200.

22 Fehrenbach A, Fehrenbach H, Wittwer T, Ochs M, Wahlers T, Richter J. Evaluation of pulmonary edema: stereological versus gravimetrical analysis. Eur Surg Res 2001; 33: 270-278.

23 Kraemer HJ, Schmidt R, Guenther A, Becker G, Suzuki Y, Seeger W. ELISA technique for quantification of surfactant protein B (SP-B) in bronchoalveolar lavage fluid. Am J Respir Crit Care Med 1995; 152: 1540-1544.

24 Schmidt R, Steinhilber W, Ruppert C, et al. An ELISA technique for quantification of surfactant apoprotein (SP)-C in bronchoalveolar lavage fluid. Am J Respir Crit Care Med 2002; 65: 470-474.

25 Bligh EG, Dyer WJ. A rapid method for total lipid extraction and purfication. Can J Biochem Physiol 1959; 37: 911-917.

26 Rouser GS, Fleischer S, Yamamoto A. Two-dimensional thin layer chromatographic separation of polar lipids and determination of phospholipids by phosphorus analysis of spots. Lipids 1970; 5: 494-496. 
27 Enhorning G. Pulsating bubble technique for evaluating pulmonary surfactant. J Appl Physiol 1977; 43: 198-203.

28 Hohlfeld JM, Hoymann HG, Tschernig T, Fehrenbach A, Krug N, Fehrenbach $\mathrm{H}$. Keratinocyte growth factor transiently alters pulmonary function in rats. J Appl Physiol 2004; 96: 704-710.
29 Fehrenbach A, Bube C, Hohlfeld JM, et al. Surfactant homeostasis is maintained in vivo during keratinocyte growth factor-induced rat lung type II cell hyperplasia. Am J Respir Crit Care Med 2003; 167: 1264-1270.

30 Griese M. Pulmonary surfactant in health and human lung diseases: state of the art. Eur Respir J 1999; 13: 1455-1476. 